\title{
Análisis de la indisponibilidad de la flota de transporte escolar de la Universidad Técnica de Manabí
}

\author{
Analysis of unavailability in the school transport park of the University \\ Technic from Manabi \\ Jorge Tamayo Mendoza ${ }^{1}$, Joel Guillén García ${ }^{1}$, Leisis Villar Ledo², Armando Díaz \\ CONCEPCión $^{2}$ y Jorge Baste GonZalez ${ }^{2}$
}

\section{RESUMEN}

El trabajo fue desarrollado en el área de mantenimiento de la flota de transporte escolar de la Universidad Técnica de Manabí debido al impacto negativo en el proceso docente que están tienen la pérdida de disponibilidad de los ómnibus y el aumento de la insatisfacción de los clientes, tanto los alumnos como los trabajadores, así como las afectaciones al proceso docente que trae consigo esta indisponibilidad. Se realiza un análisis de los factores que más están incidiendo en la función mantenimiento y en la pérdida de disponibilidad, que es el objetivo de la investigación. Para esto se tomo como referencia los datos del año 2014. Para este análisis se emplearon herramientas como el análisis de Pareto e Ishikawa, arrojando que del total de la flota, 3 de los buses son los de mayor impacto en la indisponibilidad, la misma estuvo sobre un valor de $29 \%$. Los resultados obtenidos validan la hipótesis que existe afectaciones en la disponibilidad de la flota de trasporte escolar y que los mismos responden fundamentalmente a problemas organizativos.

Palabras clave: disponibilidad; flota de transporte; mantenimiento; satisfacción clientes.

\section{ABSTRACT}

This paper was developed in the area of maintenance of school transport Park of the Technical University of Manabi. The unavailability of the bus caused the dissatisfaction of students, workers and the involvement of the teaching process. The analysis of the factors that are affecting the maintenance function and loss of availability will be the target of the research, these data for the year 2014. For this analysis were taken as reference tools they were used as the Pareto analysis and Ishikawa, showing that 3 of

1 Universidad Técnica de Manabí. Portoviejo, Ecuador.

2 Instituto Superior Politécnico José Antonio Echevarría. CUJAE. La Habana, Cuba. 
the buses from the total fleet were of major impact in the non disponibility. The value of the non disponibility was over than $29 \%$. The results validate the hypothesis that there effects on the availability of school transport Park and that they respond mainly to organizational problems.

Keywords: maintenance; availability; transport fleet; customer satisfaction.

\section{ICHIKLLACHAW}

Kay urya rurakashqa Manabiipa Hatun Yachay Wasi Tiknikachaw tsaychaw yachakuq wamrakuna apaq kaarrukunash mana alli kayan, tsayraykurshi yachatsikuqkunapa uryan mana allitsu. Tsaychawmi rikapashqa kashqa hina imakunash kayan maa tsay kananpaq, tsay kashqa 2014 watachawmi. Kay uryachaw iñishikashqa Pareto e Ishikawa rikapakuyninkunam, tsaywanmi yarqamushqa: llapan kaarrukunata rikapaarir, kima kaqkunam mana alli uryayan. Tsay chanintsashqa kashqa 29\%. Tsay tarikashqakunam chanintsaatsiyan hipuutisista nishqanta: mana alli kaarrukuna uryayaptinqa tanqanakun wakin Hatun Yachay Wasichaw ruraykunawanmi.

Pushaq shimikuna: Imapaqpis kaq; ayka imatapis puritsinapaq; alli katsiy; rantiqkunapa kushikuynin.

\section{INTRODUCCIÓN}

Con el decurso histórico el mantenimiento ha ido evolucionando hasta convertirse en lo que es hoy, una actividad imprescindible dentro de cualquier contexto operacional. En los sistemas educacionales esta máxima también se cumple; el fortalecimiento de un mantenimiento de calidad en la transportación escolar debe ser sobre las bases de logara un menor costo de mano de obra y otros costos asociados y la no afectación al proceso docente.

La Universidad Técnica de Manabí (UTM), en el Ecuador, fue creada el 29 de noviembre de 1952, actualmente cuenta con una planta de más de 600 docentes nombrados. Debido a este crecimiento la Universidad se vio en la necesidad de adquirir buses para el transporte de docentes, empleados y estudiantes. Los buses de la Universidad Técnica son para uso de los profesores, empleados y estudiantes que viven dentro de la ciudad de Portoviejo, Manabí, los cuales hacen cuatro recorridos en el día con rutas específicas.

La UTM cuenta con un parque automotor importante debido a su crecimiento tanto docente como de empleados y estudiantes, viéndose en la necesidad de tener un departamento de mantenimiento, que no está cumpliendo con su misión. Este incumplimiento provoca que, en la actualidad, la UTM tenga un parque automotor de 9 buses, de los cuales cuatro se encuentran en mal estado, cuatro en estado regular, y uno fue recién adquirido, pues se compró al final del año 2014. Las unidades de transporte hacen dos recorridos por la mañana y dos recorrido por las tarde, para darse mayor apoyo al cumplimiento del proceso docente. 
No se ha encontrado referencias bibliográficas de estudios realizados para este campo de investigación. Está claro que esta situación no es única de la UTM, pero se ha podido apreciar en otras investigaciones en marcos de estudios análogos, que se han empleado formas de minimizar estos problemas, muy propios de los lugares donde fueron originados, y no ha existido una propuesta para tener una forma de actuación ante esta problemática.

En la actualidad se viene confrontando problemas de indisponibilidad en la flota de transporte, fallas repetidas, la gerencia de transporte así como la dirección de la institución presentan preocupaciones con los costos asociados a este proceso que se viene incrementando. Esta indisponibilidad en la gran mayoría de las ocasiones ha conllevado al incumpliendo de los planes de transportación, afectando la llegada de los estudiantes y/o profesores, estos últimos en menor escala, afectando el proceso docente y la insatisfacción de los empleados. Conocer los problemas que más están incidiendo en esta pérdida de disponibilidad es el propósito de la presente investigación.

\section{MATERIALES Y MÉTODOS}

Se realizó una investigación descriptiva. Para la obtención de los datos se utilizó un método de revisión de órdenes de trabajo aplicando el método de análisis y síntesis. Para la realización de un análisis en cualquier proceso es necesario comenzar por conocer cuál es la situación actual y cuáles serían los elementos que más están influyendo en el problema analizado, pero para realizar este paso es necesario crear un grupo de trabajo para implementar un trabajo por tormenta de ideas y así potenciar las decisiones que se tome (Mora, 2006). Para complementar esta etapa se desarrolló un método de análisis de expertos, para calcular el coeficiente de competencia de los expertos, a partir de un instrumento con preguntas acerca de la función mantenimiento (figura 1), tomando como muestra el grupo de trabajo del Servicio de Transporte de UTM.

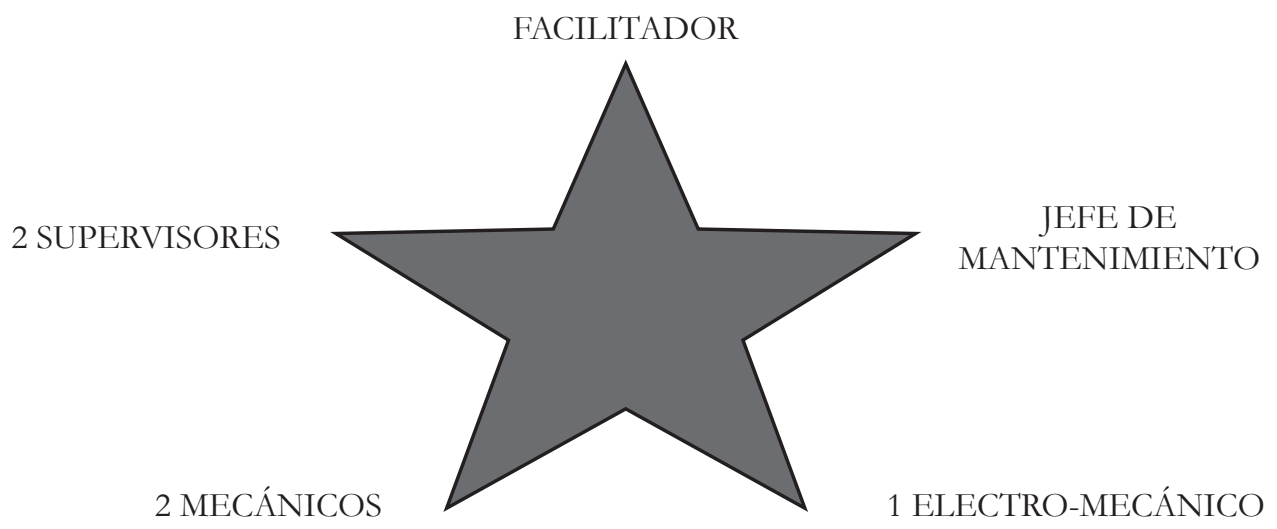

Figura 1. Integrantes del grupo natural de trabajo

Para el análisis de las causas que más están influyendo en la indisponibilidad de los ómnibus y que están afectando el cumplimento del proceso docente y el aumento de la insatisfacción de los alumnos y trabajadores se utiliza un diagrama de Ishikawa o Diagrama causa efecto (Gutiérrez, 2013). 
Esta herramienta, también llamada diagrama de causa-efecto, diagrama causal que por su estructura ha venido a llamarse también: diagrama de espina de pescado, pues consiste en una representación gráfica sencilla en la que puede verse de manera relacional una especie de espina central, que es una línea en el plano horizontal, representando el problema a analizar, que se escribe a su derecha; en esta investigación será disponibilidad de la flota de transporte de la UTM.

Para la identificación de las causas más probables los expertos encerraron en un círculo la(s) causa(s) más probable(s) seleccionadas por el equipo o marcarla con un asterisco y le dieron un nivel de impacto (Díaz et al., 2012).

También se realizó un análisis de la disponibilidad (Hayes, 2005; Bonet, 2011; Rodríguez, 2009; García, 2005), partiendo del levantamiento de las fallas ocurridas en los activos a través de la ecuación 1 y de la indisponibilidad, ecuación 2, el año 2014 de la flota de transporte de la UTM.

La disponibilidad es la probabilidad de que un activo realice la función asignada cuando se requiere de ella durante un período determinado, asimismo depende de cuán frecuente se producen los fallos y de cuánto tiempo se requiere para corregir el fallo (Viego et al., 2015).

El cálculo de la disponibilidad está basado en la distribución de fallas y la distribución de tiempo de reparación y muestra la probabilidad de que el equipo esté operando satisfactoriamente en el momento en que sea requerido después del comienzo de su operación.

$$
D I S P=\frac{\sum(H C A L-H R M N)}{\sum H C A L} * 100
$$

HCAL: Horas calendario (Tiempo que estuvo en funcionamiento el equipo).

HRMN: Horas de reparación por mantenimiento (tiempo que estuvo detenido el equipo por mantenimiento).

$$
\text { Indispon. }=1-\text { Disp. }
$$

Se aplicó un análisis Pareto (Gutiérrez, 2013) que es una técnica que separa los «pocos vitales» de los «muchos triviales» permitiendo la toma de decisiones al equipo de trabajo (Tavare, 2000; Sexto, 2004).

\section{RESULTADOS}

La flota de transporte de la UTM está compuesta por 5 vehículos ligeros destinados, fundamentalmente, a la transportación de las autoridades, y por 9 buses destinados a la transportación de alumnos, profesores y personal administrativo. Este grupo de buses es el que será objeto de la investigación, ya que la afectación en sus unidades incide negativamente en el cumplimento del proceso docente y la satisfacción de los clientes. En la tabla 1 se muestran una descripción de los mismos. 
Tabla 1. Descripción general de los buses de la UTM

\begin{tabular}{ccccc}
\hline N $^{\text {de Unidad }}$ & Marca & Año & Uso & Asientos \\
\hline International & International & 2015 & Estudiantes y profesores & 45 \\
\# 13 MEC 343 & JAC & 2007 & Estudiantes y profesores & 40 \\
International MTI -1227 & International & 2014 & Estudiantes y profesores & 45 \\
International MEI -1226 & International & 2014 & Estudiantes y profesores & 45 \\
International NUEVO & International & 2015 & Estudiantes y profesores & 45 \\
\# 1 MEC -0184 & HINO & 2001 & Estudiantes y profesores & 45 \\
\#5 MEI- 1022 & ISUSU & 1991 & Estudiantes y profesores & 45 \\
\# 12 MEC 346 & JAC & 2007 & Estudiantes y profesores & 45 \\
\#8 MEC -0182 & CHEVROLET & 2002 & Estudiantes y profesores & 40 \\
\hline
\end{tabular}

\section{Elección de los expertos}

Como paso inicial en la elección de los expertos se utilizó la herramienta de Kendal donde los resultados del Ka o coeficiente de argumentación obtenidos se muestran en la tabla 2 .

Tabla 2. Resultados del coeficiente de argumentación, Ka

\begin{tabular}{lccccccccc}
\hline \multicolumn{1}{c}{ Fuentes de argumentación } & E1 & E2 & E3 & E4 & E5 & E6 & E7 & E8 & E9 \\
\hline Análisis teóricos realizados por usted. & 0,3 & 0,3 & 0,2 & 0,3 & 0,2 & 0,1 & 0,3 & 0,1 & 0,3 \\
$\begin{array}{l}\text { Su experiencia obtenida. } \\
\text { Libros de autores nacionales que ha } \\
\text { leído. }\end{array}$ & 0,5 & 0,5 & 0,5 & 0,5 & 0,5 & 0,2 & 0,5 & 0,2 & 0,5 \\
$\begin{array}{l}\text { Libros de autores internacionales que ha } \\
\text { leído. }\end{array}$ & 0,05 & 0,05 & 0,05 & 0,04 & 0,04 & 0,03 & 0,05 & 0,04 & 0,05 \\
Su propio conocimiento del problema. & 0,03 & 0,04 & 0,03 & 0,04 & 0,04 & 0,03 & 0,03 & 0,03 & 0,05 \\
Su propia intuición. & 0,05 & 0,05 & 0,05 & 0,05 & 0,04 & 0,03 & 0,04 & 0,04 & 0,04 \\
\multicolumn{1}{c}{ TOTAL } & 0,05 & 0,05 & 0,05 & 0,05 & 0,05 & 0,03 & 0,05 & 0,04 & 0,05 \\
& 0,98 & 0,99 & 0,88 & 0,98 & 0,87 & 0,42 & 0,97 & 0,45 & 0,99 \\
\hline
\end{tabular}

De los resultados de la tabla 2, se aprecia que los especialistas 6 y 8 tienen un bajo coeficiente de argumentación, respecto a los demás especialistas.

A continuación, en la tabla 3 se muestra los resultados del cálculo del Coeficiente de Conocimiento Kc. 
Tabla 3. Cálculo del Coeficiente de Conocimiento Kc

\begin{tabular}{|c|c|c|c|c|c|c|c|c|c|}
\hline \multicolumn{10}{|c|}{ ITEM / EXPERTO } \\
\hline & E1 & E2 & E3 & $\mathrm{E} 4$ & E5 & E6 & E7 & E8 & E9 \\
\hline 1 & 10 & 9 & 9 & 10 & 10 & 7 & 9 & 7 & 10 \\
\hline 2 & 10 & 10 & 10 & 9 & 9 & 5 & 10 & 5 & 10 \\
\hline 3 & 10 & 7 & 7 & 10 & 10 & 6 & 7 & 6 & 10 \\
\hline 4 & 10 & 9 & 10 & 10 & 10 & 7 & 10 & 5 & 10 \\
\hline 5 & 8 & 9 & 8 & 10 & 9 & 2 & 8 & 2 & 8 \\
\hline 6 & 7 & 7 & 7 & 9 & 7 & 3 & 7 & 3 & 9 \\
\hline 7 & 7 & 9 & 9 & 10 & 6 & 5 & 9 & 5 & 7 \\
\hline 8 & 8 & 8 & 8 & 6 & 8 & 6 & 8 & 6 & 8 \\
\hline 9 & 5 & 7 & 6 & 10 & 5 & 8 & 6 & 6 & 9 \\
\hline 10 & 7 & 8 & 7 & 8 & 7 & 3 & 7 & 3 & 9 \\
\hline 11 & 9 & 10 & 10 & 7 & 10 & 8 & 10 & 8 & 8 \\
\hline 12 & 5 & 6 & 6 & 10 & 5 & 6 & 6 & 6 & 10 \\
\hline 13 & 5 & 8 & 7 & 5 & 10 & 2 & 7 & 2 & 8 \\
\hline 14 & 9 & 10 & 9 & 9 & 9 & 4 & 9 & 4 & 9 \\
\hline 15 & 10 & 9 & 10 & 10 & 10 & 9 & 10 & 7 & 10 \\
\hline Total & 7,2 & 7,4 & 7,4 & 7,9 & 7,5 & 4,8 & 7,34 & 4,5 & 8,1 \\
\hline Kc & 0,72 & 0,76 & 0,74 & 0,8 & 0,75 & 0,49 & 0,74 & 0,45 & 0.81 \\
\hline
\end{tabular}

De los resultados de la tabla 3, se aprecia que los especialistas 6 y 8 tienen un bajo coeficiente de conocimiento, respecto a los demás especialistas; comportamiento similar al que ocurrió con el coeficiente de argumentación.

En la tabla 4 se muestran los resultados del cálculo del coeficiente de experticia.

Tabla 4. Cálculo del coeficiente de experticia

\begin{tabular}{cccccccccc}
\hline & E1 & E2 & E3 & E4 & E5 & E6 & E7 & E8 & E9 \\
\hline $\mathrm{K}=0,5^{*}(\mathrm{Kc}+\mathrm{Ka})$ & 0,85 & 0,873 & 0,809 & 0,889 & 0,81 & 0,453 & 0,854 & 0,45 & 0,9 \\
\hline
\end{tabular}

De los resultados obtenidos se desecha como expertos a los especialistas 6 y 8 , por lo que el trabajo continúa con 7 expertos que apoyarán las decisiones en adelante.

2. Análisis de las causas que inciden en la pérdida de disponibilidad

Para este análisis se utilizó como herramienta la de causa - efecto o análisis de Ishikawa. Se debe aclarar que las causas y subcausas fueron analizadas y tomadas por consenso con el Grupo Natural de Trabajo. En la figura 2 se expone los resultados. 


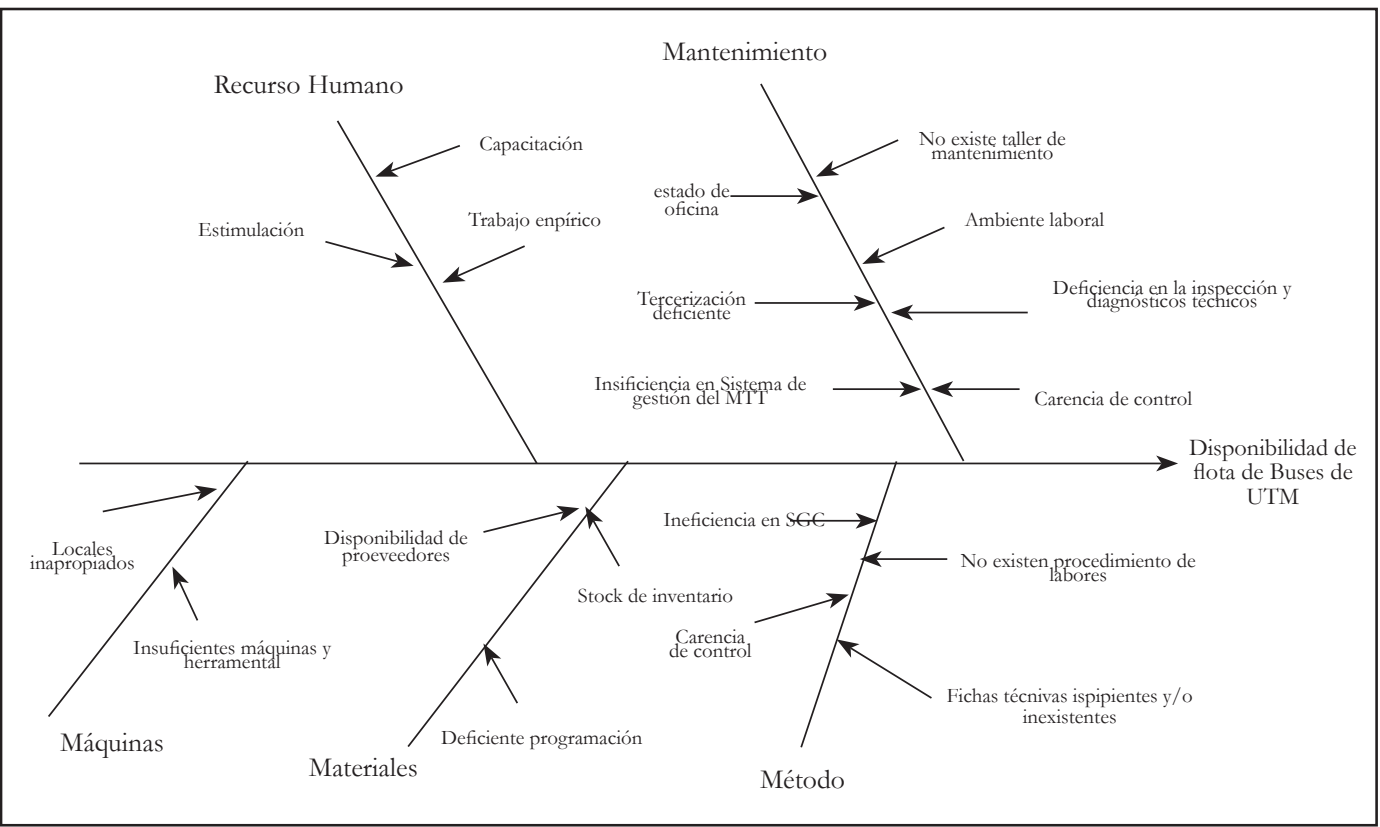

Figura 2. Diagrama causa efecto

En la tabla 5 se expone de forma resumida los resultados que aparecen en la figura 2 donde también se muestra los valores de mayor impacto obtenidos en las variables analizadas a partir del análisis del Grupo Natural de Trabajo y usando como herramienta la tormenta de ideas y para verter los resultados se utilizó el método de tipo «post-it» donde de forma consensuada se llegó a los resultados planteados.

Tabla 5. Resultados del análisis causa efecto

\begin{tabular}{llc}
\hline Causas & \multicolumn{1}{c}{ Subcausas } & Nivel de impacto \\
\hline \multirow{2}{*}{$\begin{array}{c}\text { Recursos } \\
\text { Humanos }\end{array}$} & Poca capacitación al personal que labora & $80 \%$ \\
& Trabajo empírico & $75 \%$ \\
& No existe sistema de calidad implementado & $85 \%$ \\
Métodos & No existe procedimientos para las labores & $80 \%$ \\
& Carencia de control y manejo de indicadores de satisfacción & $70 \%$ \\
& Fichas técnicas insipientes y en muchos casos inexistentes & $85 \%$ \\
& Stock de piezas de repuestos insuficientes & \\
Materiales & Falta de programación en las actividades & \\
& Poca disponibilidad de proveedores & $70 \%$
\end{tabular}




\begin{tabular}{ll} 
Oficina en mal estado y poco ergonómica & $65 \%$ \\
Inexistencia de taller de mantenimiento & $90 \%$ \\
Inconformidad con el ambiente laboral & $75 \%$ \\
Trabajos de tercerización muy lentos y engorrosos & $70 \%$ \\
Mantenimiento & El trabajo del técnico es solo para verificar o valorar el daño \\
del vehículo o buses & $90 \%$ \\
No existe sistema de gestión de mantenimiento implementado & $70 \%$ \\
Carencia de control y manejos de indicadores de manteni- & \\
\hline
\end{tabular}

\section{Análisis de la indisponibilidad de la flota}

En la tabla 6 se expone los tiempos perdidos de cada bus donde al tomar como 16 horas las que el mismo tiene que estar disponible, se puede observar en la columna de la derecha los días indisponibles de forma redondeada, la cantidad de días hábiles a tener en cuenta es 283 días al año. Estos datos fueron obtenidos de los reportes del año 2014.

Tabla 6. Resumen del tiempo perdido por buses en el año 2014

\begin{tabular}{clcc}
\hline No. & \multicolumn{1}{c}{ Tipo de bus } & $\begin{array}{c}\text { Horas } \\
\text { perdidas }\end{array}$ & $\begin{array}{c}\text { Viajes } \\
\text { indisponibles }\end{array}$ \\
\hline 1 & BUS INTERNACIONAL & 660 & 41 \\
2 & BUS \# 13 MEC 343 & 1725 & 108 \\
3 & $\begin{array}{l}\text { BUS INTERNACIONAL } \\
\text { MTI -1227 }\end{array}$ & 11265 & 704 \\
& BUS INTERNACIONAL & 11535 & 721 \\
& MEI -1226 & & \\
5 & BUS INTERNACIONAL & 240 & 15 \\
& NUEVO & 570 & 36 \\
7 & BUS \# 1 MEC -0184 & 15630 & 977 \\
8 & BUS \#5 MEI- 1022 & 2505 & 157 \\
9 & BUS \# 12 MEC 346 & 5370 & 336 \\
& $\quad$ Total & 49500 & 3095 \\
\hline
\end{tabular}

A partir de los resultados mostrados en la tabla 6 , se observa que los buses 3,4 y 7 tienen un mayor impacto en la indisponibilidad de los viajes, seguido por el bus $9 \mathrm{y}$ en menor medida por los buses 2 y 8 . En la figura 3 y 4 se observa los resultados de la aplicación de la herramienta Pareto a la flota en las variables horas perdidas y viajes dejados de realizar. 


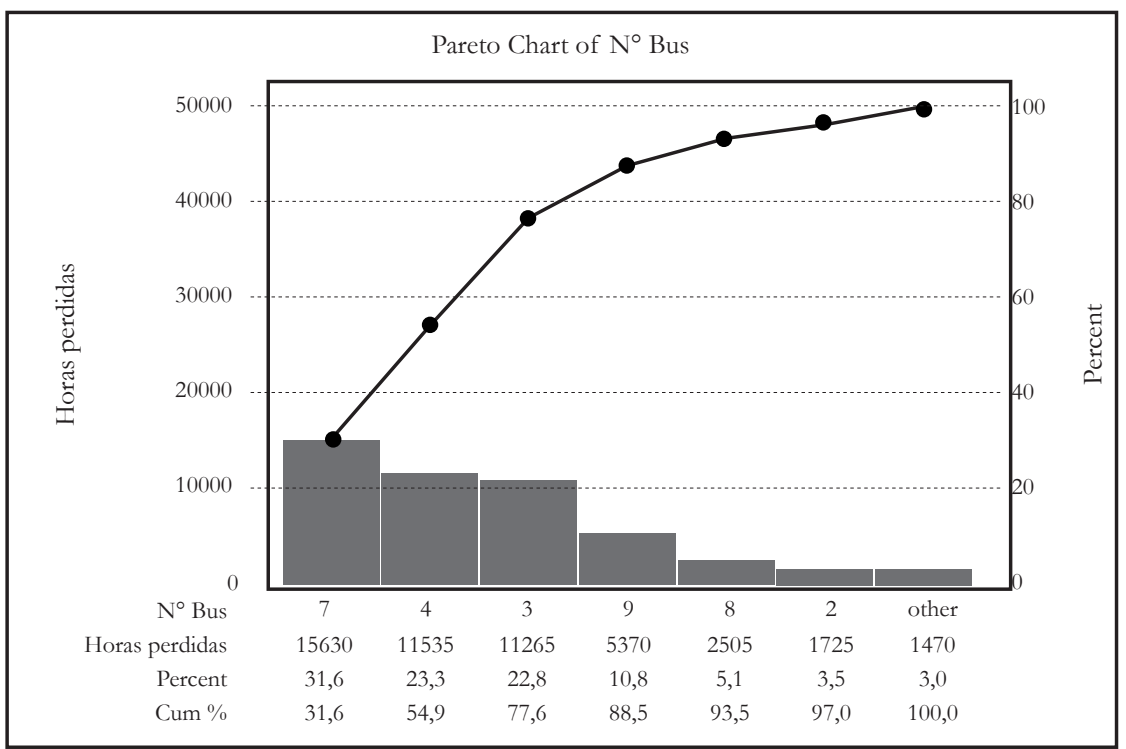

Figura 3. Resultados atendiendo a horas perdidas

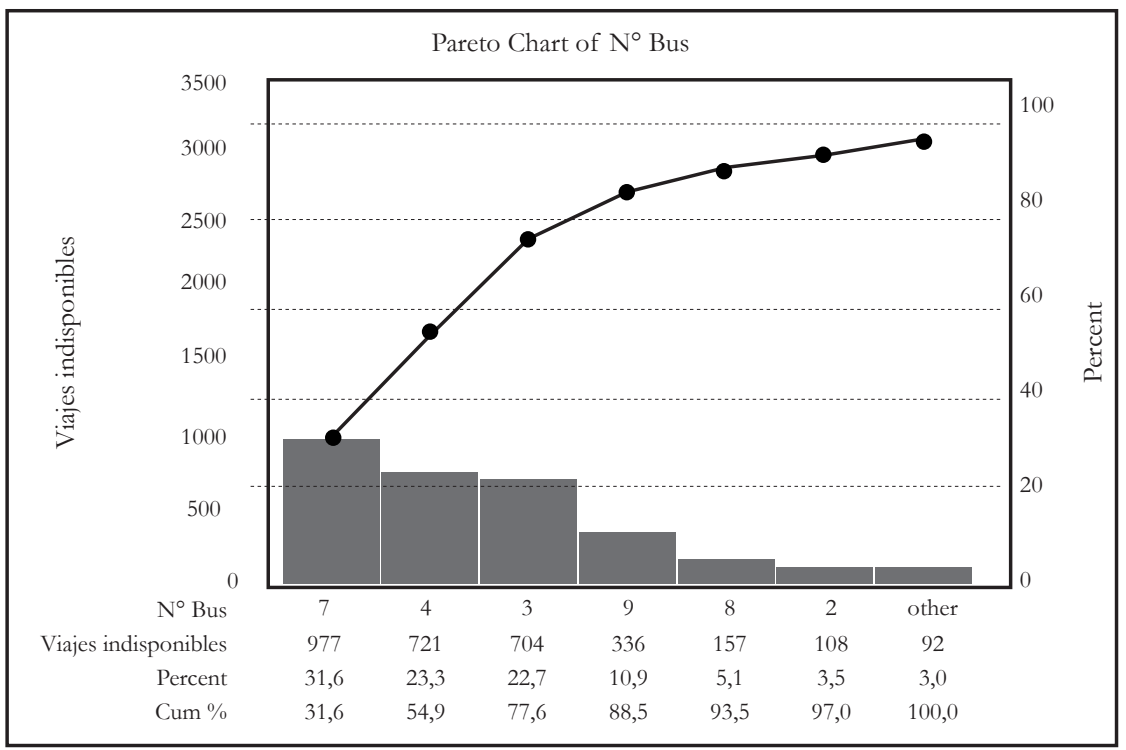

Figura 4. Resultados atendiendo a viajes indisponibles

Del resultado de la figura 3 se obtiene que los buses BUS \#5 MEI-1022, BUS INTERNACIONAL MEI-1226, BUS INTERNACIONAL MTI-1227 representan el 80\% del problema analizado, lo que se corrobora en la figura 4.

4. Análisis de la indisponibilidad de los buses

Tomando como base las fórmulas 1 y 2 así como los valores del año 2014 reflejados en la tabla 6 se obtiene los valores de disponibilidad e indisponibilidad que se muestran en la tabla 7 . 
Tabla 7. Resumen de la disponibilidad e indisponibilidad por buses y total en el año 2014

\begin{tabular}{clcc}
\hline No. & \multicolumn{1}{c}{ Tipo de bus } & Disponibilidad \% & Indisponibilidad \% \\
\hline 1 & BUS INTERNACIONAL & 93,2 & 6,8 \\
2 & BUS \# 13 MEC 343 & 84,0 & 16,0 \\
& BUS INTERNACIONAL & & 55,4 \\
3 & MTI -1227 & 44,6 & \\
& BUS INTERNACIONAL & & 56,0 \\
4 & MEI -1226 & 44,0 & \\
& BUS INTERNACIONAL & & 2,6 \\
5 & NUEVO & 97,4 & 6,0 \\
6 & BUS \# 1 MEC -0184 & 94,0 & 63,3 \\
7 & BUS \#5 MEI- 1022 & 36,7 & 21,7 \\
8 & BUS \# 12 MEC 346 & 78,3 & 37,3 \\
9 & BUS \#8 MEC -0182 & 62,7 & 29,5 \\
\hline & Total & 70,5 & \\
\hline
\end{tabular}

\section{DISCUSIÓN}

A partir de los resultados que se muestran en la figura 1 y la tabla 5 , se puede destacar que la mayoría de las causas que están influyendo están referidas a problemas de organización, aunque los mismos son problemas particulares de este campo en otros análisis, ejemplo en (Bonet, 2011; Rodríguez, 2009) el estudio estaba dirigido a flotas de trasporte público y no obstante se evidenciaban problemas similares. Dentro de estas se puede concluir que las que más están incidiendo en la pérdida de disponibilidad de los Buses en la UTM están enfocados al recurso humano, afectado fundamentalmente por la poca oportunidad de capacitación y estimulación, los materiales, debido al déficit de piezas de repuesto para los buses y al mantenimiento, causa que presenta el mayor grupo de subcausas, donde el no contar con locales adecuados y los sistemas que rijan la actividad da al traste con el cumplimiento del objetivo de esta área.

De los análisis Pareto que se muestran en las figuras 3 y 4, se pudo demostrar que tres buses, el BUS \#5 MEI-1022, el BUS INTERNACIONAL MEI-1226 y el BUS INTERNACIONAL MTI-1227, son los más críticos, al ser los que afectan en aproximadamente el $80 \%$ de la problemática, tanto por horas perdidas como por viajes indisponibles.

El valor de indisponibilidad calculado de los buses fue de un $25 \%$ dado que los mismos no están sometidos a una explotación agresiva, y comparando este valor con los resultados mostrados en la tabla 7 , se puede concluir que el valor es no adecuado y por tanto incide en el cumplimiento de su función fundamental que es, la de garantizar el cumplimento de proceso docente con no afectaciones por traslado y la insatisfacción de los clientes de forma general. 


\section{CONCLUSIONES}

Se analizaron las características actuales del mantenimiento en la UTM, la cual arroja problemas que inciden en afectaciones al proceso docente y a la satisfacción de los clientes.

Se determina que entre los elementos que más afectan, están los problemas de mantenimiento, recursos humanos y materiales.

La indisponibilidad de la flota es de un 29\% por debajo de estándares para estos tipos de flota.

\section{AGRADECIMIENTOS}

Los autores agradecemos el apoyo del Centro de Estudios de Ingeniería y Mantenimiento CEIM de la Facultad de Ingeniería Mecánica de la CUJAE, Cuba, por su apoyo y a la UTM por la atención y acceso a la información.

\section{REFERENCIAS BIBLIOGRÁFICAS}

Bonet, Carlos. 2011. Fiabilidad Aplicada al Transporte. La Habana: Editorial Federico Engels.

Díaz, Armando y otros. 2012. «Propuesta de un modelo para el análisis de criticidad en plantas de productos biológicos». Revista Ingeniería Mecánica. Vol. 15, No 1. ISSN 1815-5944. La Habana.: Instituto Superior Politécnico José Antonio Echeverría. 34-43.

García, Oliverio. 2005. «El Análisis Causa Raíz, Estrategia de Confiabilidad Operacional». Colombia: Conferencia y Exhibición, Reliability World Latín América. <http:// equiposrotativos.galeon.com/Analisis.pdf> [Consulta: 20-10-2015].

Gutiérrez, Humberto y De La Vara, Román. 2013. Control estadístico de calidady seis sigma. México: Mc Graw Hill.

Hayes, Bob. 2005. Como medir la satisfacción de cliente. Barcelona: Ediciones Gestión 2000.

Mora, Luis. 2006. Mantenimiento estratégico para empresas industriales o de servicios. Antioquia: Ultragráficas Ltda.

Rodríguez, Emilio. 2009. Establecimiento de políticas de mantenimiento a los equipos de la Sucursal Transporte de la Compañia Almacenes Universales S.A. La Habana: Instituto Superior Politécnico José Antonio Echeverría. <http://biblioteca.cujae.edu. cu:81/TesisPregrado> [Consulta: 10-06-2015].

Sexto, Luis Felipe. 2004. Nivel de Calidad Seis Sigma: Paradigma del Mantenimiento a Sistemas Críticos. Malvinas: Instituto Peruano de Mantenimiento. <http://docplayer. es /1429327-La-creatividad-en-accion-triz-six-sigma-y-rcm-entre-el-retoinnovador-y-la-necesidad-industrial-1.html> [Consulta: 20-06-2015]. 
Tavare, Lourival. 2000. Administración moderna del mantenimiento. Sao Pablo: Datastream Systems do Brasil. <http://es.slideshare.Net/CarlosAlbertoZiga/ administración-moderna-de-mantenimiento-lourival-tavares $>$ [Consulta: 20-062015].

Viego, Néstor et al. 2015. «Estudio de confiablidad operacional como soporte al mantenimiento aeronáutico en Cuba». Revistas Ingenieras. Vol. XVIII, No. 662015. ISSN 1405-0676. México: Universidad Autónoma de Nuevo León. 6-12.

Fecha de recepción: 28 de noviembre 2015

Fecha de aceptación: 01 de diciembre 2015

\section{Correspondencia}

Jorge Tamayo Mendoza

jtamayo@utm.edu.ec 